\title{
Global dynamics of a vector-borne disease model with latency and saturating incidence rate
}

\author{
S.M. Ashrafur Rahman \\ University of Khulna, Khulna-9208, Bangladesh \\ Email: arahman_ku@yahoo.com
}

Copyright (C2014 S.M. Ashrafur Rahman. This is an open access article distributed under the Creative Commons Attribution License, which permits unrestricted use, distribution, and reproduction in any medium, provided the original work is properly cited.

\begin{abstract}
This paper deals with a vector-borne disease model containing latency and nonlinear incidence rates. Global analysis is completely determined by suitable Lyapunov functionals. We explicitely determine the basic reproduction number and find that if this number is less than one then disease dies out, but if the number is larger than one, the disease causing strain become endemic. The study shows that the latency delay explicitely influence the disease persistence.
\end{abstract}

Keywords: Latency, saturating incidence, basic reproduction number, global attractivity, Lyapunov functionals.

\section{Introduction}

Cooke [5] presented a mathematical model to describe the dynamics of a communicable disease through a vector population based on the scenario below. Human beings are divided into three classes: the susceptible class with its population denoted by $S(t)$, the infective class with its population denoted by $I(t)$, and the removed class with its population denoted by $R(t)$. The susceptible vectors are infected by infectious human individuals. It was assumed in [5] that it takes an infected vector $\tau$ time units to become infectious. Being infectious, the vectors then infect susceptible human individuals. Denote the populations of susceptible and infectious vectors at time $t$ by $V_{S}(t)$ and $V_{I}(t)$ respectively. As a vector population is usually quite large, it was also simply assumed in [5] that $V_{I}(t)$ is proportional to $I(t-\tau)$. Adopting the mass action infection mechanism $\hat{\beta} V_{I}(t) S(t)$, the force of infection for human beings at time $t$ is then given by

$\hat{\beta} V_{I}(t) S(t) \approx \beta I(t-\tau) S(t)$.

This leads to the following model of delay differential equations

$$
\left\{\begin{array}{l}
\dot{S}(t)=\Lambda-\mu S(t)-\beta S(t) I(t-\tau), \\
\dot{I}(t)=\beta S(t) I(t-\tau)-(\mu+\gamma) I(t), \\
\dot{R}(t)=\gamma I(t)-\mu R(t)
\end{array}\right.
$$

where $R$ is the population of the removed class. For more biological explanations and the mathematical results for this model, see $[1,12,16]$. 
Table 1: Description of variables and parameters of model (4).

\begin{tabular}{ll}
\hline Parameter & Description \\
\hline$\Lambda$ & Recruitment of individuals \\
$\frac{1}{\mu}$ & Life expectancy \\
$\beta$ & Transmission coefficient of susceptibles to infectives \\
$\frac{1}{\gamma}$ & Average infected period of invectives \\
$\alpha$ & Saturation level \\
$\mu_{1}$ & Combination of infection induced death rate and natural death \\
& rate of infectives \\
\hline
\end{tabular}

The mass action law for infection is the simplest mechanism since it assumes a linear incidence rate $g(I)=\beta I$. Motivated by Capasso [3], many authors (see, e.g., [4, 9, 17, 20]) have used a saturating incidence rate defined by

$h(I)=\frac{\beta I}{1+\delta I}$,

where $\delta \geq 0$ determines the saturation levels when the infectious population is large. When $\delta=0$, this reduces to the mass action incidence rate. Thus the saturated incidence rate is a generalization of mass action incidence rate and is more reasonable as it reflects the crowding effect of the infective individuals. On the hand, delay has an impact on the mortality of vector population. In this paper, we modified the model (2) to introduce delay induced death rate as well as saturating incidence.

The rest of this paper is organized as follows. In Section 2, we formulate a vector-borne disease model based on those in $[5,17,14]$. Equilibria and the basic reproduction number of the model are discussed in Section 3. Section 4 deals with global stability of the equilibria. Numerical simulations are given in Section 5. Finally, Section 6 offers some concluding remarks and discussion.

\section{A Vector-borne disease model}

The model we present here is a straight forward modification of (2) by incorporating saturation incidence and delay induced death rate of vector. To proceed, we denote by $S$, and $I$ the sub-populations of susceptible and infective classes respectively. Let $R$ still be the population of the removed class. Following the discussion in the introduction, the vector's sub-populations can be omitted from the equations by including delays in the infectious classes of human beings. In order to incorporate delay induced death rate and saturation incidence rate, we modified the equation (2). Subsequently, the model equations of the dynamics of such a vector-borne disease, assuming a saturating incidence rate, is then governed by the following system of delay differential equations:

$$
\left\{\begin{array}{l}
\dot{S}(t)=\Lambda-\mu S(t)-\frac{\beta e^{-d \tau} S(t) I(t-\tau)}{1+\alpha e^{-d \tau} I(t-\tau)}, \\
\dot{I}(t)=\frac{\beta e^{-d \tau} S(t) I(t-\tau)}{1+\alpha e^{-d \tau} I(t-\tau)}-\left(\mu_{1}+\gamma\right) I(t), \\
\dot{R}(t)=\gamma I(t)-\mu R(t) .
\end{array}\right.
$$

The parameters in the model are summarized in Table 1. To avoid excessive brackets and notational ambiguity, we use the convention: $I_{\tau}=I(t-\tau)$.

For biological reasons, we need to pose the following conditions on initial values of the unknowns:

$S(0)>0$ and $I(\theta)=\phi(\theta) \geq 0$

where

$\phi \in C\left([-\tau, 0], \mathfrak{R}_{+}\right)$. 


\section{Basic properties of the model}

Applying the standard argument, one can easily show that for initial functions satisfying (5)-(6), the system (4) has a unique solution with all components being non-negative. Adding all equations of (4), the total population $N=S+I+R$ satisfies

$\dot{N} \leq \Lambda-\mu N$.

Here we have used the reasonable assumption

$\mu \leq \mu_{1}$,

meaning the mortality rates for infectious individuals are not less than that of the susceptible individuals. By comparison theorem, it follows that

$$
\limsup _{t \rightarrow \infty} N(t) \leq \frac{\Lambda}{\mu}
$$

This suggests confining ourselves to the biologically feasible region

$\Gamma=\left\{(S, I, R): S, I, R \geq 0, S+I+R \leq \frac{\Lambda}{\mu}\right\}$.

Since $\mathrm{R}$ is decoupled in (4), it is sufficient and reasonable to analyze the reduced two dimensional system

$$
\left\{\begin{array}{l}
\dot{S}(t)=\Lambda-\mu S(t)-\frac{\beta e^{-d \tau} S(t) I(t-\tau)}{1+\alpha e^{-d \tau} I(t-\tau)}, \\
\dot{I}(t)=\frac{\beta e^{-d \tau} S(t) I(t-\tau)}{1+\alpha e^{-d \tau} I(t-\tau)}-\left(\mu_{1}+\gamma\right) I(t) .
\end{array}\right.
$$

Corresponding to (7), we only need to consider for (8) the following set

$\Gamma_{1}=\left\{(S, I): S, I \geq 0, S+I \leq \frac{\Lambda}{\mu}\right\}$

which is obviously positively invariant for (8).

\subsection{Steady states}

The system has a disease-free equilibrium (DFE) $E_{0}$ given by,

$E_{0}=\left(S_{0}, 0\right), \quad$ with $S_{0}=\frac{\Lambda}{\mu}$.

There is a possible co-existence equilibrium (all components are positive), defined by

$E^{*}=\left(S^{*}, I^{*}\right)=\left(\frac{\Lambda \alpha e^{-d \tau}+\mu_{1}+\gamma}{\beta e^{-d \tau}+\alpha \mu e^{-d \tau}}, \frac{\Lambda \beta e^{-d \tau}-\mu\left(\mu_{1}+\gamma\right)}{\left(\mu_{1}+\gamma\right)\left(\beta e^{-d \tau}+\alpha \mu e^{-d \tau}\right)}\right)$.

It is readily seen that the co-existence equilibrium is biologically significant if and only if

$\mathfrak{R}_{0}:=\frac{\Lambda \beta e^{-d \tau}}{\mu\left(\mu_{1}+\gamma\right)}>1, \quad i=1,2$

where $\mathfrak{R}_{0}$ is the basic reproduction number of the model.

By Theorem 2 in [19], we have the following theorem relating the stability/instability of $E_{0}$ to the value of $\Re_{0}$.

Theorem 3.1 The DFE $E_{0}$ is asymptotically stable, if $\mathfrak{R}_{0}<1$; and it becomes unstable if $\mathfrak{R}_{0}>1$. 


\section{Global stability analysis}

In this section, we discuss global stability of each of the equilibria. To this end, we apply Lyapunov functionals similar to those recently used by $[8,10,14]$. Such Lyapunov functionals take advantages of the properties of the function

$g(x)=x-1-\ln (x)$,

which is positive in $\mathcal{R}_{+}$except at $x=1$ where it vanishes. In the following theorems we show that each of the equilibria exhibits global stability under some threshold conditions. We begin with the DFE $E_{0}$.

Theorem 4.1 The DFE $E_{0}$ is globally asymptotically stable if $\mathfrak{R}_{0}<1$.

Proof: Consider the Lyapunov functional

$V(S, I)=S_{0} g\left(\frac{S}{S_{0}}\right)+I+\beta S_{0} \int_{-\tau}^{0} I(t+\theta) d \theta$.

Obviously, $V$ is non-negative in the positive cone $\left.\Omega=\mathcal{R}_{+} \times C\left([-\tau, 0], \mathfrak{R}_{+}\right), \mathfrak{R}_{+}\right)$and attains zero at $E_{0}$. We need to show $\dot{V}$ is negative definite. Differentiating $\mathrm{V}$ along the trajectories of (8), we obtain

$$
\begin{aligned}
& \dot{V}=\left(1-\frac{S^{0}}{S}\right) \dot{S}+\dot{I}+\beta e^{-d \tau} S_{0}\left(I-I_{\tau}\right), \\
&=\left(1-\frac{S^{0}}{S}\right)\left(\Lambda-\mu S-\frac{\beta e^{-d \tau} S I_{\tau}}{1+\alpha e^{-d \tau} I_{\tau}}\right)+\left(\frac{\beta e^{-d \tau} S I_{\tau}}{1+\alpha e^{-d \tau} I_{\tau}}-\left(\mu_{1}+\gamma\right) I\right) \\
& \quad+\beta e^{-d \tau} S_{0}\left(I-I_{\tau}\right), \\
&=-\mu\left(S-S_{0}\right)^{2}-\frac{\beta e^{-d \tau} S I_{\tau}}{1+\alpha e^{-d \tau} I_{\tau}}+\frac{\beta e^{-d \tau} S_{0} I_{\tau}}{1+\alpha e^{-d \tau} I_{\tau}} \\
& \quad+\left(\frac{\beta e^{-d \tau} S I_{\tau}}{1+\alpha e^{-d \tau} I_{\tau}}-\left(\mu_{1}+\gamma\right) I\right)+\beta e^{-d \tau} S_{0}\left(I-I_{\tau}\right), \\
&=-\mu\left(S-S_{0}\right)^{2}-\frac{\beta e^{-d \tau} S_{0} I_{\tau}^{2}}{1+\alpha e^{-d \tau} I_{\tau}}-\left(\mu_{1}+\gamma\right) I+\beta e^{-d \tau} S_{0} I \\
&=-\mu\left(S-S_{0}\right)^{2}-\frac{\beta e^{-d \tau} S_{0} I_{\tau}^{2}}{1+\alpha e^{-d \tau} I_{\tau}}+\left(\mu_{1}+\gamma\right) I\left(\frac{\beta e^{-d \tau} S_{0}}{\left(\mu_{1}+\gamma\right)}-1\right), \\
&=-\mu\left(S-S_{0}\right)^{2}-\frac{\beta e^{-d \tau} \Lambda I_{\tau}^{2}}{1+\alpha e^{-d \tau} I_{\tau}}+\mu\left(\mu_{1}+\gamma\right) I\left(\Re_{\mathrm{o}}-1\right) .
\end{aligned}
$$

Therefore, $\dot{V} \leq 0$ if $\mathfrak{R}_{0}<1$ with equality holding only at $E_{0}$. By Theorem 5.3.1 of [7], the solutions approach $\mathcal{M}$, the largest invariant set of $\left\{\frac{d V}{d t}=0\right\}$. Since $\frac{d V}{d t}$ is zero only at $E_{0}, \mathcal{M}=\left\{E_{0}\right\}$ is a singleton set. Thus, the equilibrium $E_{0}$ is globally attractive if $\Re_{\mathfrak{o}}<1$. Considering this fact along with Theorem 3.1, we can conclude that the DFE is indeed globally asymptotically stable if $\Re_{0}<1$.

Theorem 4.2 Let the co-existence equilibrium $E^{*}$ exist (i.e. $\mathfrak{R}_{\mathrm{o}}>1$ ). Then $E^{*}$ is globally attractive.

Proof: Consider the Lyapunov functional

$V=\frac{1}{\beta e^{-d \tau} f(\bar{I})} V_{S}+\frac{\bar{I}}{\beta e^{-d \tau} \bar{S} f(\bar{I})} V_{I}+\bar{V}_{I}$

where

$$
\begin{aligned}
V_{S} & =g\left(\frac{S}{\bar{S}}\right) \\
V_{I} & =g\left(\frac{I}{\bar{I}}\right) \\
\bar{V}_{I} & =\int_{0}^{\tau} g\left(\frac{I(t-s)}{\bar{I}}\right) d s,
\end{aligned}
$$


with

$$
f(x)=\frac{x}{1+\alpha e^{-d \tau} x} .
$$

By the properties of $g$ function, it is easy to see that the Lyapunov functional $\mathrm{V}$ is non-negative in positive cone $\Omega$ and attains zero at $E^{*}$. That is $\mathrm{V}$ is positive definite. We need to show $\dot{V}$ is negative definite. Differentiating $\mathrm{V}$ along the solution of (8), we obtain

$\dot{V}=\frac{1}{\beta e^{-d \tau} f(\bar{I})} \dot{V}_{S}+\frac{\bar{I}}{\beta e^{-d \tau} \bar{S} f(\bar{I})} \dot{V}_{I}+\dot{\bar{V}}_{I}$

Calculating each derivatives separately, we obtain

$$
\begin{aligned}
\dot{V}_{S} & =\frac{1}{\bar{S}}\left(1-\frac{\bar{S}}{S}\right) \dot{S} \\
& =\frac{1}{\bar{S}}\left(1-\frac{\bar{S}}{S}\right)\left(\Lambda-\mu S-\beta e^{-d \tau} S f\left(I_{\tau}\right)\right) .
\end{aligned}
$$

Using the equation of (8) for the equilibrium $E^{*}$ and rearranging the terms, we get

$$
\begin{aligned}
\dot{V}_{S} & =\frac{1}{\bar{S}}\left(1-\frac{\bar{S}}{S}\right)\left(\mu \bar{S}+\beta e^{-d \tau} \bar{S} f(\bar{I})-\mu S-\beta e^{-d \tau} S f\left(I_{\tau}\right)\right), \\
& =\frac{1}{\bar{S}}\left(1-\frac{\bar{S}}{S}\right)\left\{\mu(\bar{S}-S)+\beta e^{-d \tau}\left(\bar{S} f(\bar{I})-S f\left(I_{\tau}\right)\right)\right\}, \\
& =-\frac{\mu}{S \bar{S}}(S-\bar{S})^{2}+\beta e^{-d \tau} f(\bar{I})\left(1-\frac{\bar{S}}{S}\right)\left(1-\frac{S}{\bar{S}} \frac{f\left(I_{\tau}\right)}{f(\bar{I})}\right) .
\end{aligned}
$$

We define

$x=\frac{S}{\bar{S}}, \quad y=\frac{I}{\bar{I}}, \quad z=\frac{I_{\tau}}{\bar{I}}$,

and

$F(z)=\frac{f\left(\bar{I}_{1} z\right)}{f(\bar{I})}=\frac{f\left(I_{\tau}\right)}{f(\bar{I})}$

then we can write

$$
\dot{V}_{S}=-\frac{\mu}{S \bar{S}}(S-\bar{S})^{2}+\beta e^{-d \tau} f(\bar{I})\left(1-\frac{1}{x}-x F(z)+F(z)\right) .
$$

Similarly, evaluating $\dot{V}_{I}, \dot{\bar{V}}_{I}$ and substituting in (12), the detail calculations are omitted here, we obtain

$$
\begin{aligned}
\dot{V} & =\left[-\frac{\mu}{\beta e^{-d \tau} f(\bar{I})} \frac{(S-\bar{S})^{2}}{S \bar{S}}+\left(1-\frac{1}{x}-\ln x\right)+\left(1-\frac{x F(z)}{y}+\ln \left(\frac{x F(z)}{y}\right)\right)\right. \\
& +F(z)-z+\ln z-\ln F(z)] .
\end{aligned}
$$

Following [14] together with the fact that the first term in the above expression is negative,we can show that $\dot{V} \leq 0$ with equality holding only at $E^{*}$. Thus, the solutions approach $\mathcal{M}$, the largest invariant set of $\left\{\frac{d V}{d t}=0\right\}[7]$. Since $\frac{d V}{d t}$ is zero only at $E^{*}, \mathcal{M}=\left\{E^{*}\right\}$ is a singleton set. Thus, the equilibrium $E^{*}$ is globally attractive.

\section{Numerical simulations}

In this section, we simulate the model for some parameter values. The parameters are chosen so that it can illustrate some vital aspects of the model which have been also confirmed by analytical conclusions. In Figure 1, we see that the disease free equilibrium is a global attractor where latencey, $\tau$, is relatively large. As $\tau$ decreases the basic reproduction number increases that leads the endemic equilibrium to become endemic(Figure 2). Therefore, the latency has a vital impact on the disease dynamics. 


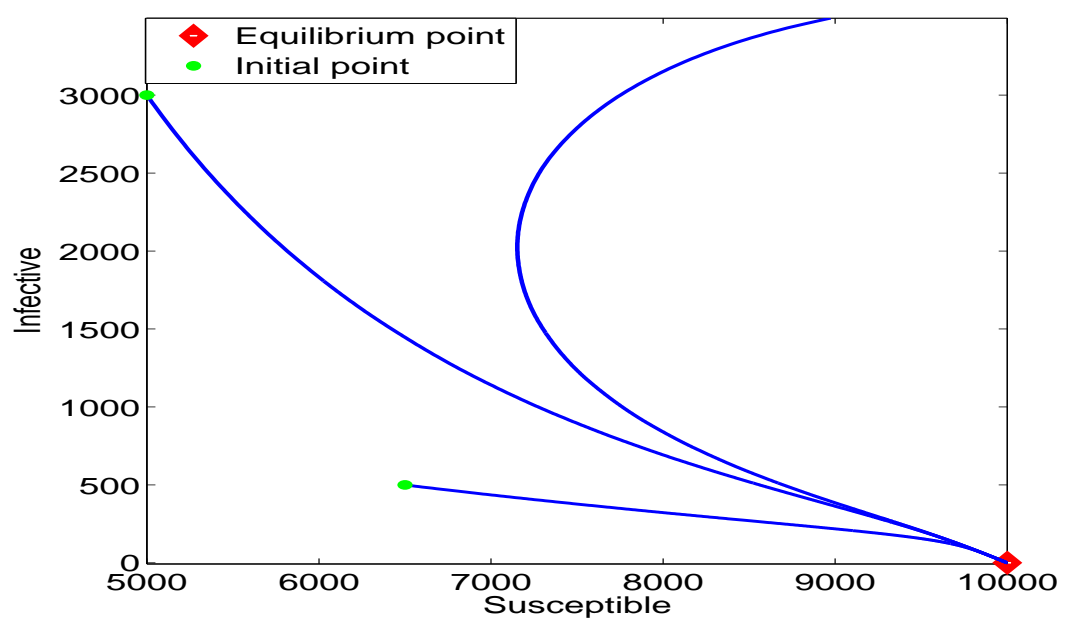

Figure 1: Global stable endemic equilibrium. Parameter values are $\beta=0.0042 ; \gamma=0.021 ; \mu=0.02 ; \mu_{1}=0.02 ; \tau=$ 7.0 ; and $\Lambda=200$, giving $\mathfrak{R}_{0}=0.93$.

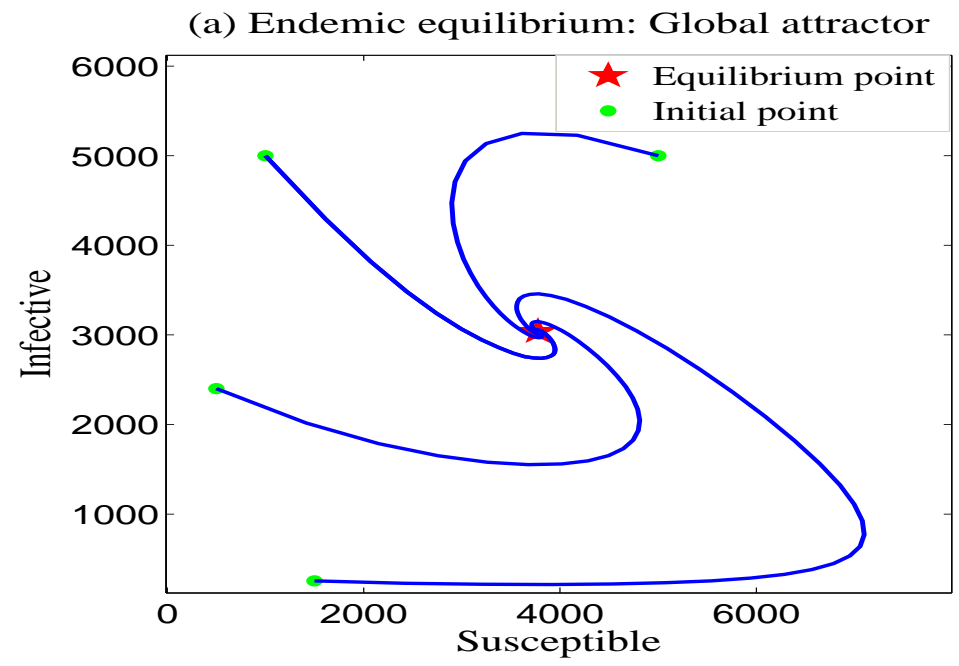

Figure 2: Global stable endemic equilibrium. Parameter values are $\beta=0.0042 ; \gamma=0.021 ; \mu=0.02 ; \mu_{1}=0.02 ;$ and $\Lambda=$ 200, giving $\mathfrak{R}_{0}=2.67$.

\section{Discussion}

In this paper, we have formulated a vector-borne disease model with latency and saturating incidence rate. We have shown that if the basic reproduction number $\mathfrak{R}_{0}$ is less than one, the disease dies out from the population. However, if $\Re_{0}>1$ the disease persists.

The existence of equilibria and the value of reproduction number(10) depend, explicitly, on the latency delay. As $\tau$ increases the basic reproduction number decreases. If the latency is long enough so that $\mathfrak{R}_{0}<1$, then the endemic equilibrium can not exist and disease will disappear shortly. The saturation level also has an impact on the level of endemic equilibrium. The $\mathrm{S}$ component of endemic equilibrium increases, while I component decreases as saturation level increases.

The global nature of the dynamics of this model is completely determined by selecting suitable Lyapunov functionals. The numerical simulations also support this claim. It is observed that one of the equilibria is always globally attractive. That is if an equilibrium is locally stable then is so globally.

Finally, our analysis evince that both delay and saturating incidence have an effect on the dynamics of infection and disease. 


\section{References}

[1] F. Beretta, V. Capasso, F. Rinaldi, Global stability results for a generalized Lotka-Volterra system with distributed delays, J. Math. Biol., 26(1988), 661-688.

[2] M.C. Bruce, M.R. Galindki, J.W. Barnwell, C.A. Donnelly, M. Walmsley, M.P. Alpers, D. Walliker and K.P. Day, Vaccination in disease models with antibody-dependent enhancement, J. Math. Biosci., 211(1988),265-281.

[3] V. Capasso, and G. Serio, A generalization of the Kermack-Mckendrick deterministic epidemic model, Math. Biosci., 42(1978), 41-61.

[4] L. Cai, X. Li and J. Yu, Analysis of a delayed HIV/AIDS epidemic model with saturation incidence, J. Appl. Math. Comput., 27(2008),365-377.

[5] K.L. Cooke, Stability analysis for a vector disease model, Rocky Mountain J. Math., 9(1979), 31-42.

[6] O. Diekmann, J.S.P. Heesterbeek and J.A.J. Metz, On the definition and the computation of the basic reproduction ratio R_0 in models for infectious diseases in heterogeneous populations, J. Math. Biol., 28(1990), 365-382.

[7] J. Hale and S.V. Lunel, Introduction to functional Differential Equations, Springer-Verlag, New York, 1993.

[8] H. Guo, M.Y. Li and Z. Shuai, A graph-theoretic approach to the method of global Lyapunov functions, Proc. Amer. Math. Soc., 136(2008), 2793-2802.

[9] J. Hou and Z. Teng, Continuous and Impulsive Vaccination of SEIR epidemic models with saturation incidence rates, Mathematics and Computers in Simulation, 79(2009), 3038-3054.

[10] A. Korobeinikov, and P.K. Maini, A Lyapunov function and global properties for SIR and SEIR epidemiological models with nonlinear incidence, Math. Biosci. Eng., 1(2004), 57-30.

[11] J.P. LaSalle, The Stability of Dynamical Systems, SIAM, Philadephia, 1976.

[12] W. Ma, Y. Takeuchi, T. Hara and E. Beretta,Permanence of an epidemic model with distributed time delays, Tohoku Math. J., 54(2002), 581-591.

[13] M.J. Mackinnon and A.F. Read,Virulence in malaria: an evolutionary viewpoint, Phil. Trans. R. Soc. Lond. B, 359(2004), 965-986.

[14] C.C. McCluskey, Global stability for an SIR epidemic model with delay and nonlinear incidence, Nonlin. Anal. RWA, 11(2010), 3106-3109.

[15] N. Mideo and T. Day, On the evolution of reproductive restraint in malaria, Proc. R. Soc. B, 275(2008), $1217-1224$.

[16] J.M. Tchuenche, A. Nwagwo and R. Levins, Global behaviour of an SIR epidemic model with time delay, Math. Meth. Appl. Sci., 30(2007), 733-749.

[17] R. Xu and X. Ma, Global stabailty of a SIR epidemic model with nonlinear incidence rate and time delay, Nonlin. Anal. $R W A, \mathbf{1 0}(2009), 3175-3189$.

[18] C. Wongsrichanalai, C.K. Murray, M. Gray, R.S. Miller, P. Mcdaniel, W.J. Liao, A.L. Picard and A. J. Magill, Coinfection with malaria and leptospirosis, Am. J. Trop. Med. Hyg., 68(2003), 583-585.

[19] P. van den Driessche and J. Watmough, Reproduction Numbers and subthreshold endemic equilibria for compartmental models of disease transmission, Math. Biosci., 180(2002), 29-48.

[20] Y. Zhou, D. Xiao and Y. Li, Bifurcations of an epidemic model with non-monotonic incidence rate of saturated mass action, Chaos, Solitons and Fractals, 32(2007), 1903-1915. 